\title{
The Association Between RNF213, MMP3 , and Moyamoya Disease: Screening the Full Exons of Susceptibility Genes in Families with Moyamoya Disease
}

\section{Yilei Xiao}

Liaocheng People's Hospital

\section{Donghu Yu}

Wuhan University Zhongnan Hospital

Weidong Liu

Liaocheng People's Hospital

Jiheng Hao

Liaocheng People's Hospital

\section{Yuejiu Pang}

Shandong Provincial Hospital

\section{Qunlong Jiang}

Liaocheng People's Hospital

\section{Min Zhang}

Shandong Provincial Hospital

\section{Xingbang Wang}

Qilu Hospital of Shandong University Qingdao

\section{Xueyuan Li \\ Liaocheng People's Hospital}

\section{Zixin Guo}

Wuhan University Zhongnan Hospital

\section{Xiaoping Liu}

Wuhan University Zhongnan Hospital

Jie Yao

Wuhan Bojie Biomedical Science and Technology CO., LTD

\section{Liyong Zhang}

Liaocheng People's Hospital

\section{Zhaogang Dong}

Qilu Hospital of Shandong University Qingdao Department of Endocrinology

Jiyue Wang ( $\nabla$ wangjiyue002100@163.com )

Liaocheng People's Hospital https://orcid.org/0000-0003-2938-5365 
Keywords: Moyamoya disease, family, whole exome sequencing, CRISPR-Cas12a

Posted Date: June 1st, 2021

DOI: https://doi.org/10.21203/rs.3.rs-546805/v1

License: (c) (i) This work is licensed under a Creative Commons Attribution 4.0 International License. Read Full License 


\section{Abstract}

Background: In this study, we aimed to investigate the association between polymorphisms in the RNF213 and MMP3 genes and Moyamoya disease (MMD) and to screen susceptibility genes for families with MMD.

Methods: The mutation of RNF213 gene (rs112735431,rs148731719) and MMP3 gene rs3025058 were detected by first generation sequencing and CRISPR-Cas12a. The genetic susceptibility genes of MMD families samples were studied by whole exome sequencing (WES).

Results: The application of CRISPR-Cas12a and Sanger sequencing identified rs112735431 and rs148731719 mutations in the RNF213 gene, and an insertion mutation in the MMP3 gene (rs3025058) with significant regional differences $(P<0.05)$. Additionally, we identified 100\% agreement between CRISPR-Cas12a and Sanger sequencing with regards to the detection of these mutations in samples, thus indicating that CRISPR-Cas $12 a$ can be used for the detection of MMD mutations. Next, we carried out whole exon screening for family members with MMD and identified multiple recessive genes associated with genetic disease. Notably, the Titin (TTN) gene (rs771533925, rs559712998 and rs72677250) was significantly associated with point mutations. Screening of the Exome Aggregation Consortium (EXAC) database identified significant differences between these populations with respect to frequency. PROVEANヌnvariant Feature Transform (SIFT) and PolyPhen algorithms further demonstrated that rs771533925 and rs72677250 were potentially damaging in three databases, rs559712998 was considered to be tolerated in above mentioned databases. Scale Invariant Feature Transform (SIFT) and PolyPhen algorithms further demonstrated that rs771533925 and rs72677250 were potentially damaging by all three databases and that rs559712998 was considered to be tolerated in above mentioned databases. Gene Ontology (GO) analysis demonstrated that the target of TTN is involved in many important biological processes. The screening of 50 sporadic MMD samples failed to identify any mutations, thus indicating that single nucleotide polymorphism (SNP) mutation sites (rs771533925, rs559712998, and rs72677250) within the TTN gene may play an important role in the inheritance of MMD.

Conclusions: In summary, our study investigated susceptibility genes for MMD from multiple perspectives and found that CRISPR-Cas12a technology can efficiently detect MMD mutation sites. Our findings provide a theoretical basis for future investigations of the molecular mechanisms that underlie MMD.

\section{Introduction}

Moyamoya disease (MMD) is a chronic progressive, cerebrovascular, and occlusive disease of unknown etiology. MMD was first reported and named by Suzuki in 1969 [1]. MMD is associated with a high disability rate and is characterized by stenosis and occlusion of the end of the internal carotid artery and the beginning of the Circle of Willis and the normal vascular network in the brain base. MMD is more common in people living in East Asian countries compared to the Western Hemisphere. The prevalence of MMD was previously reported to be 3.92/100 000 in Nanjing, China [2]. Studies have shown that the age of MMD onset shows a bimodal pattern, predominantly affecting those aged $0-10$ years and 30-40 years [3]. Ring finger protein 213 (RNF213), an phenotype-related gene that is located at 17q25 and expressed in endothelial cells, was the first pathogenic gene to be associated with MMD [4]. Two mutations within the RNF213 gene (rs112735431 and rs148731719) are known to be associated with the pathogenesis of MMD in Chinese patients [5-7]. In 2010, researchers discovered that the -1171 locus of the MMP3 gene in Chinese Han patients was closely related to the onset of MMD [8]; this work also represented the first research on susceptibility genes in China [8]. At present, the genes and 
polymorphic loci that are closely related to the pathogenesis of MMD are still not fully defined. Therefore, it is vital that we identify and determine the genetic factors related to MMD to study the pathogenesis of MMD and develop new methods to prevent and treat this disease.

Researchers have shown that $10 \%-15 \%$ of patients with MMD have a family history and that the risk of MMD in family members is 30 to 40 -fold higher than in the general population [9]. Over recent years, a large number of studies have confirmed the existence of genetic susceptibility and that genetic factors play a major role in the pathogenesis of MMD [9-12]. The incidence of MMD varies significantly between different races and shows a greater association with Asian races, particularly Japan, South Korea, and China [13]. In families with strong heritability, MMD often presents with an autosomal dominant inheritance pattern [14]. For example, genetic analysis of MMD families in Algeria revealed a recessive inheritance pattern indicating the existence of an $\mathrm{x}$ linked inheritance [15]. In Japan, the prevalence of familial MMD disease is 10\%-15\% [16]. In South Korea, familial cases account for $11.9 \%$ of the total number of MMD cases [17]. MMD has been detected in most parts of China, although the incidence is relatively higher in Shandong, Henan, and Hebei and other places; studies have shown that $6-10 \%$ of MMD cases are associated with a family history of the disease [18].

The CRISPR-Cas $[19,20]$ system is an important immune defense system of Archaea and bacteria against virus and plasmid infection [21]. Cas12a (cpf1) is a new type of programmable DNA enzyme found in the CRISPR system and contains an RuvC domain and a specific nuclease domain [22]. In the presence of specific directing crRNA, Cas12a recognizes the target DNA specified by the crRNA sequence to form a complex and activates specific cleavage. Some studies have found that Cas12a also has the ability to cut non-target DNA following cleavage of the target DNA [23, 24]. CRISPR-Cas biology has revolutionized the field of molecular diagnostics for various diseases; this is because this system has an extremely high sensitivity for the detection of nucleic acids [25].

In this study, we analyzed susceptibility genes in families with MMD using whole exome sequencing (WES) to provide a basis for the prevention and treatment of MMD. We also investigated the genetic distribution and clinical characteristics of MMD to provide guidance for early diagnosis and treatment that is both effective and precise.

Previous research on complex diseases has mostly focused on genome-wide association studies (GWAS). However, the GWAS method has many drawbacks. For example, GWAS mostly identifies disease-related gene loci in intronic or intergenic regions and rarely in functional or coding regions; furthermore, many of these have a minor allele frequency (MAF) $>5 \%$. An increasing body of evidence now supports the fact that complex diseases are caused by rare variants with a MAF $<5 \%[26,27]$. In addition, the size of the entire genome, and the timeconsuming nature of this technique, create significant limitations. With the development of high-throughput sequencing technology, WES has been increasingly used in the study of Mendelian diseases and complex diseases. WES refers to a genome analysis method that uses sequence capture technology to capture and enrich DNA in exonic regions of the whole genome for high-throughput sequencing. The human exome region accounts for only $1 \%$ of the entire genomic sequence, but about $85 \%$ of known pathogenic mutations are located in this region [28]. WES has several advantages over whole genome sequencing. First, the cost of WES is relatively low. Second, the sequencing time is shorter due to fewer sequences, thus improving efficiency. Third, WES can be used for sequencing integration analysis regardless of sample size, whether the data is derived from the same family or not. Finally, WES can determine candidate genes more accurately and is therefore more convenient for 
subsequent screening and the identification of gene function. In this study, we used WES to sequence samples from families with MMD and analyzed the WES data to identify susceptibility genes. Our aim was to provide a basis for the screening and diagnosis of MMD.

In this study, we aimed to to explore the efficacy of CRISPR-Cas12a technology to detect mutations. while to screen patients with MMD for the RNF213 and MMP3 genes, to explore the genetic distribution and clinical phenotype of MMD. Finally, we used WES technology to analyze samples provided by families with MMD. In future, disease-related mutation loci could be screened to identify related genes and reveal the molecular mechanisms responsible for MMD.

\section{Methods}

\section{Collection of clinical samples}

We recruited patients with no previous or current medical history ( $\geq 18$ years-of-age) with a clinical diagnosis of MMD that had been confirmed by transcranial computed tomography (CT), Magnetic resonance imaging (MRI), or DigitalSubtractionAngiography (DSA) imaging; medical history and various clinical judgments. Fasting samples of venous blood were collected from all patients. We also collected fasting blood samples from healthy control subjects during the same period. All subjects, or their guardians, provided a signed consent form prior to entering the trial.

\section{Primer design and preparation of crRNA}

Wild-type and mutant templates were designed with reference to the known mutation detection loci for the RNF213 gene. Amplimers and crRNAs were then designed for the known mutation regions and oligonucleotides (crDNA) were synthesized. crDNA and cr-T7-F were mixed and boiled for 10 minutes, then the double-stranded transcription template was prepared by natural cooling. The transcription template was then incubated for 16 hours at $37^{\circ} \mathrm{C}$ under enzymatic-free conditions using the HiScribe T7 Quick High Yield RNA Synthesis Kit (NEB, Ipswich, USA). After the completion of the reaction, $2 \mu$ l of DNase 1(TianGen, Beijing, China) was added to eliminate unreacted template in the system; the desired crRNA was then purified. Wild-type and mutant template sequences, amplimers, and crDNAs, were synthesized by Tianyi Huiyuan Biotechnology Co., Ltd. (Table S1).

\section{Validation of the CRISPR-Cas12a fluorescence detection system}

Fncas12a uses 5'-KYTV-3' as protospacer adjacent moti (PAM), compared with Ascas12a and Lbcas12a, it provides more target sequence options [29], therefore, Fncas12a was chose as the detection protein. In brief, 50ng of template DNA was added into the detection reagent mixture containing $0.75 \mu \mathrm{M}$ crRNA, $1.5 \mu \mathrm{M}$ Fncas $12 \mathrm{a}$, a $50 \mathrm{pm}$ fluorescent probe, and $3 \mu \mathrm{l}$ of NEBuffer 3.1 (NEB, Ipswich, USA). A total volume of 50 $\mu$ l was then reacted at $37^{\circ} \mathrm{C}$ for $1 \mathrm{~h}$ prior to fluorescence quantification. All reactions were carried out at $37-42^{\circ} \mathrm{C}$.

\section{Clinical sample testing}


Following the separation of plasma, the venous blood samples were used to extract DNA. Then, polymerase chain reaction (PCR) was performed using specific primers, $50 \mathrm{ng}$ of DNA as the template, and the following cycle conditions: $95^{\circ} \mathrm{C}$ for $5 \mathrm{~min} ; 30$ cycles of $95^{\circ} \mathrm{C}$ for $3 \mathrm{~min} ; 56^{\circ} \mathrm{C}$ for $10 \mathrm{~s}$, and $72^{\circ} \mathrm{C}$ for $20 \mathrm{~s}$; followed by $72^{\circ} \mathrm{C}$ for 5

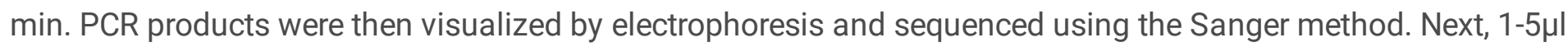
of amplified product was used for CRISPR-Cas12a fluorescence detection.

\section{Collection and selection of samples for WES}

We collected 12 samples from two families with clinical manifestations of the MMD phenotype; the samples were collected from Liaocheng People's Hospital Center (Table 1). Twelve family members with a history of MMD were subjected to WES. The sequencing process is depicted as a flow chart in Figure S1. This study was approved by the ethics committee of Liaocheng City, Shandong Province. Informed consent for DNA analysis was obtained from patients in line with local institutional review board requirements at the time of collection.

\begin{tabular}{|lll|}
\hline \multicolumn{2}{|c|}{$\begin{array}{c}\text { Table 1 } \\
\text { Sample information }\end{array}$} \\
\hline Specimen No. & Sex (male/female) & Patient or Normal (P: Patient; N: Normal) \\
\hline A1 & F & P \\
A2 & F & P \\
A3 & M & N \\
A4 & M & N \\
\hline A5 & F & N \\
\hline A6 & F & N \\
\hline A7 & M & N \\
\hline B1 & F & P \\
\hline B2 & M & N \\
\hline B3 & M & N \\
\hline B4 & M & N \\
\hline B5 & F & P \\
\hline
\end{tabular}

\section{Library construction of WES}

For each sample, the DNA extracted from peripheral blood was fragmented to an average size of 180 - 280bp and subjected to DNA library creation using established Illumina paired-end protocols. The Agilent SureSelect Human All ExonV6 Kit (Agilent Technologies, Santa Clara, CA, USA) was used for exome capture according to the manufacturer's instructions. The Illumina NovaSeq 6000 platform (Illumina Inc., San Diego, CA, USA) was utilized 
for genomic DNA sequencing in Novogene Bioinformatics Technology Co., Ltd (Beijing, China) to generate 150-bp paired-end reads with a minimum coverage of $10 \times$ for $~ 99 \%$ of the genome (mean coverage of 100x).

\section{Data analysis of WES}

After sequencing, base-call file conversions and demultiplexing were performed with bcl2fastq software (Illumina). The resulting fastq data were submitted to in-house quality control software to remove low quality reads; these were then aligned to the reference human genome (hs37d5) using the Burrows-Wheeler Aligner (bwa) [30]. Duplicate reads were marked using sambamba tools [31]. Single nucleotide variants (SNVs) and indels were identified by samtools to generate Genome VCF (gVCF) [32]. Raw calls for the SNVs and INDELs were further filtered with the following inclusion thresholds: 1 ) a read depth $>4 ; 2$ ) a root-mean-square mapping quality of covering reads that was $>30$; and 3 ) a variant quality score $>20$. Copy number variants (CNVs) were detected with CoNIFER software (Version 0.2.2) [33]. Annotation was performed using ANNOVAR (2017) [34]. Annotations included minor allele frequencies from public control data sets as well as deleteriousness and conservation scores, thus enabling further filtering and assessment of the likely pathogenicity of variants.

\section{Selection of candidate mutation loci}

Filtering for rare variants was performed as follows. First, variants with a MAF $<0.01$ in 1000 genomic data (1000g_all) [35], esp6500siv2_all, and gnomAD data (gnomAD_ALL and gnomAD_EAS); (2) only SNVs occurring in exons or splice sites (splicing junction $10 \mathrm{bp}$ ) were analyzed further since we were interested in amino acid changes; (3) synonymous single nucleotide variants (SNVs) which were not relevant to the amino acid changes predicted by dbscSNV were discarded; the small fragment non-frameshift (<10bp) indel in the repeat region defined by RepeatMasker was discarded; and (4) variations were screened according to SIFT scores [36], PolyPhen [37], MutationTaster [38] and CADD [39] software packages. Potentially deleterious variations were reserved if the scores from more than half of these four software packages supported the fact that the variations were harmful [40]. Sites (>2bp) that did not affect alternative splicing were also removed. To better predict the harmfulness of each variation, we applied the classification system put forward by the American College of Medical Genetics and Genomics (ACMG). The variations were classified as pathogenic, likely to be pathogenic, of uncertain significance, likely to be benign, or benign [41]. Depending upon various considerations (pedigree, homozygous, and compound heterozygous), variants were considered to be candidate causal variations. The relationship between the proband and the parents was estimated using the pairwise identity-by-descent (IBD) calculation in PLINK [42]. The share of IBD between the proband and parents for all trios ranged from $45 \%$ and $55 \%$.

\section{Statistical analysis}

Apply SPSS 17.0 statistical analysis software. The qualitative data and the number of cases are described in percentage, and the quantitative data are compared by independent sample t-test or analysis of variance. $P<0.05$ indicates that the difference is significant.

\section{Results}




\section{The ability of CRISPR-Cas12a to detect mutations}

Literature searches identified RNF213 as a susceptibility gene for MMD. In addition, two SNP loci, rs112735431 (R4810K) and rs148731719 (A4399T), were closely related to MMD [5-7]. crRNA was designed for two SNP point mutation loci in RNF213. The cleavage efficiency of crRNA was then verified by wild-type and mutant-target DNA; results are presented in Figure 1. The level of fluorescence derived from the mutant was twice that of the wild type, thus indicating that the CRISPR-Cas12a system constructed by crRNA was successful and could be used to detect whether there was a mutation at this locus in a clinical sample.

\section{Detection of RNF213 gene locus by CRISPR-Cas12a and Sanger}

We collected 34 samples of patients who had been clinically diagnosed with MMD and 37 healthy control samples from Liaocheng People's Hospital. In addition, 20 samples, acquired during physical examinations, were collected from Wuhan Medical Examination Center. DNA was extracted from these samples and The RNF213 gene of samples was test by CRISPR and sanger(Table 2). The coincidence rate of the test results for the rs 112735431 locus and the rs 148731719 locus was $94 \%$ and $97 \%$, respectively. The $100 \%$ coincidence between the CRISPR-Cas12a system and Sanger Sequencing with regards to detecting mutant samples indicated that the CRISPR-Cas12a detection method is accurate and has a higher sensitivity .

Table 2

Analysis of RNF213 Gene Mutation Results by CRISPR test and Sanger sequencing

\begin{tabular}{|c|c|c|c|c|c|c|c|}
\hline & \multirow[t]{2}{*}{ Genotype } & \multicolumn{3}{|c|}{ CRISPR test results } & \multicolumn{3}{|c|}{ Sanger sequencing results } \\
\hline & & $\begin{array}{l}\text { Liaocheng } \\
\text { Case } \\
\text { group } \\
(n=34)\end{array}$ & $\begin{array}{l}\text { LiaochengControl } \\
\text { group } \\
(n=37)\end{array}$ & $\begin{array}{l}\text { Wuhan } \\
\text { control } \\
\text { group } \\
(n=20)\end{array}$ & $\begin{array}{l}\text { LiaochengCase } \\
\text { group } \\
(n=34)\end{array}$ & $\begin{array}{l}\text { Liaocheng } \\
\text { control } \\
\text { group } \\
(n=37)\end{array}$ & $\begin{array}{l}\text { Wuhan } \\
\text { control } \\
\text { group } \\
(n=20)\end{array}$ \\
\hline \multirow[t]{2}{*}{ R4810K } & Mutation & $10(29.4 \%)$ & $0(0 \%)$ & $0(0 \%)$ & $8(23.5 \%)$ & $0(0 \%)$ & $0(0 \%)$ \\
\hline & wild & $24(70.6 \%)$ & $37(100 \%)$ & $\begin{array}{l}20 \\
(100 \%)\end{array}$ & $26(76.4 \%)$ & 37 (100\%) & $\begin{array}{l}20 \\
(100 \%)\end{array}$ \\
\hline \multirow[t]{2}{*}{ A4399T } & Mutation & $5(14.7 \%)$ & $3(8.1 \%)$ & $1(5 \%)$ & $4(11.8 \%)$ & $3(8.1 \%)$ & $1(5 \%)$ \\
\hline & wild & $29(85.3 \%)$ & $34(91.9 \%)$ & $\begin{array}{l}19 \\
(95 \%)\end{array}$ & $30(88.2 \%)$ & $\begin{array}{l}34 \\
(91.9 \%)\end{array}$ & $\begin{array}{l}19 \\
(95 \%)\end{array}$ \\
\hline
\end{tabular}

\section{Analysis of the correlation between gene区RNF213ロMMP3) mutations and MMD}

Analysis sanger test results of $R N F 213$,as shown in Figure 2a, there was a C>T mutation at locus rs112735431 and a $\mathrm{G}>\mathrm{A}$ mutation at locus rs148731719 of the RNF213 gene. T-tests showed that the P value for the rs112735431 locus mutation was < 0.05 when comparing between the case group and the healthy control group from the Liaocheng area. In contrast, there was no significant difference between the groups with respect to rs148731719. This indicated that the rs112735431 mutation within the RNF213 gene was significant and that the 
R4810K is a significant mutation locus for MMD in the RNF213 gene. T-tests of healthy control samples in the Liaocheng area and Wuhan area were not significant $(P>0.05)$ (Table 3a).

We identified a base insertion mutation (rs3025058) in the MMP3 gene (Figure 2b). This mutation was identified by Sanger sequencing and detected in $67.6 \%$ of the 34 patients with MMD in Shandong province, and $5.4 \%$ of the 37 controls; the detection rate was $85 \%$ in the control group (20 cases) in Wuhan City; P values were all $<0.05$, indicating statistical significance. The $1171(6 \mathrm{~A} / 6 \mathrm{~A})$ mutation in the MMP3 gene is associated with the risk of MMD; furthermore, the risk of the $(6 \mathrm{~A} / 6 \mathrm{~A})$ genotype is higher than that of the $(5 \mathrm{~A} / 6 \mathrm{~A})$ genotype (Table $3 \mathrm{~b})$. Analysis of MMP3 gene mutation results in the two regions (Shandong and Wuhan) indicated that MMD may be a regional disease; however, this needs to be verified in future research involving a larger sample size.

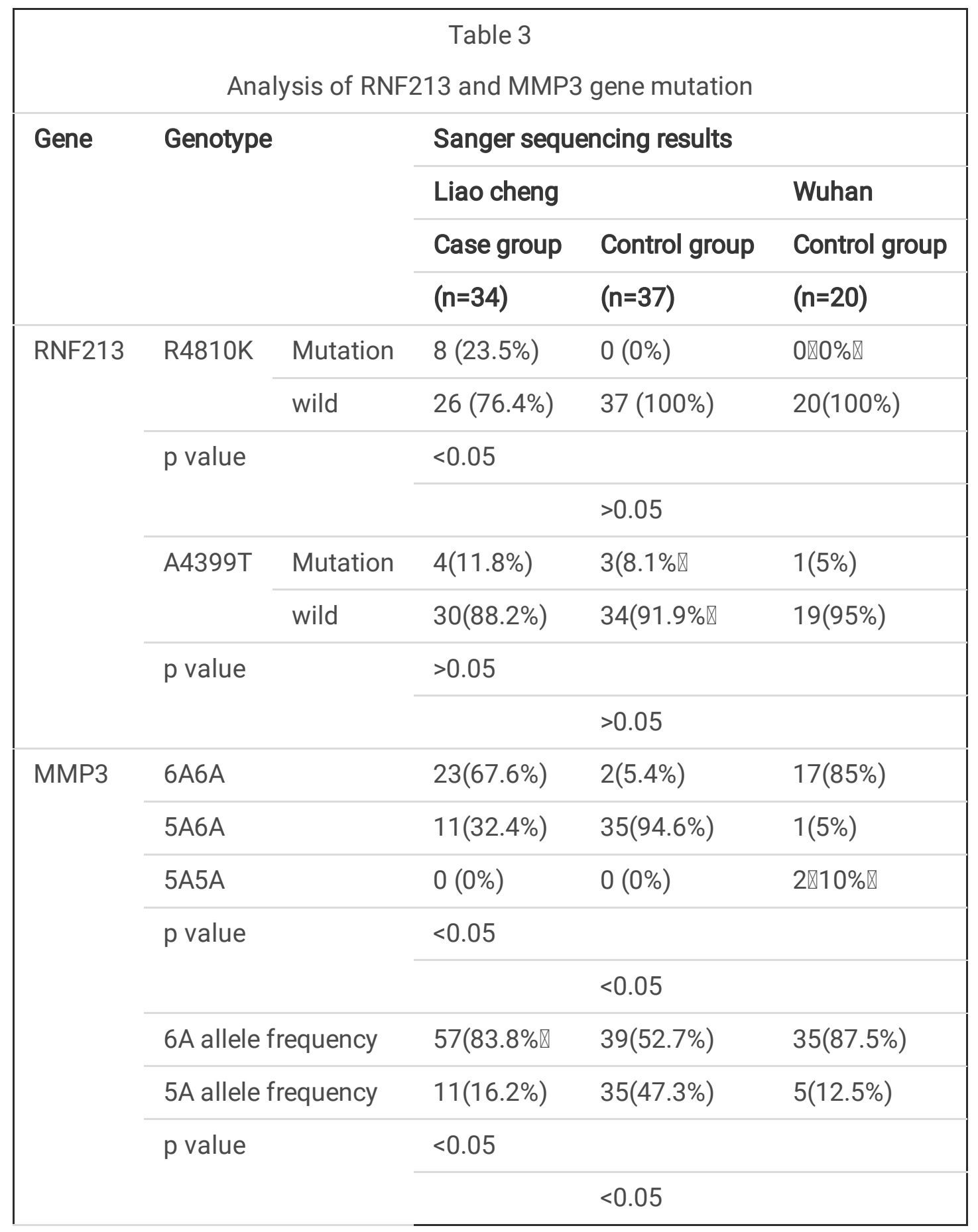




\section{WES}

In this study, draw the family maps of 12 samples and perform ct test on the patients, the results are shown in the Figure 3.The average sequencing depth of the 12 samples exceeded 100x, and the coverage of regions exceeding 10x exceeded 99\%. All exonic regions and Untranslated Regions (UTR) regions of all samples were covered effectively (Table S2). The number of SNVs and Indels obtained from each sample after data analysis is shown in Table S3.

\section{Screening for candidate pathological changes}

All SNVs and Indels were annotated to remove at least one mutation with a frequency higher than $1 \%$ from 1000g_all, esp6500siv2_all, gnomAD_ALL, and gnomAD_EAS. This practice removed diverse loci among individuals and identified rare mutations that were most likely to be pathogenic. Exonic or splicing (10bp up-and downstream) variants were retained. Synonymous SNP mutations that were not predicted by the software as affecting splicing and were located in regions that were not well conserved, were removed. The non-frameshift InDel mutation with a small fragment $(<10 \mathrm{bp})$ of the repeat region was removed. Mutation loci were screened in accordance with the scores predicted by SIFT, PolyPhen, MutationTaster, and CADD. For the locus to be retained, at least half of the scores derived by these four software packages needed to show that the locus may be harmful. Splicing mutations needed to be no further than $2 \mathrm{bp}( \pm 1-2 b p)$ from the exonic region. In addition, the dbscSNV software needed to predict that a given mutations would exert impact on splicing. Table S4 provides data related to the screening process.

\section{Screening of mutation-related genes}

Candidate loci were screened further according to the process shown in Figure 4. Recessive hereditary pathogenicity is known to be characterized by a normal parental phenotype and the presence of pathogenic loci. Offspring inherit the pathogenic homozygous locus of their parents and present as diseased individuals. Recessive genetic diseases involve the homozygous variation of genes and compound heterozygous variation. We also took X-linkage into account when we screened for recessive patterns. A recessive pattern screening refers to the preservation of a homozygous mutation in a family with a normal human heterozygous mutation or a locus without a mutation as a candidate locus if a monogenic disease is inherited in a family with a recessive pattern. Compound heterozygous pattern screening refers to the preservation of loci that are not homozygous mutations in patients and normal individuals if the monogenic disease is inherited in a recessive pattern within the family, and requires that a gene has at least two heterozygous mutation loci in the patient. The distribution of mutation loci in this gene in patients cannot be the same as that of any normal control, nor can it be a subset of mutation loci in any normal person. Using this strategy, we identified multiple recessive pathogenic genes; of these, the loci mutation-related genes were within the TTN gene (rs771533925, rs559712998 and rs72677250) (Table 4).

\section{Global population frequencies for rs72677250【rs559712998 and rs771533925}


Next, we analyzed the risk alleles (rs72677250, rs559712998 and rs771533925) in accordance with the Exome Convergence Alliance (EXAC) database [43]. We identified significant differences in frequency across the global population. The highest frequency of rs72677250 in South Asian population was 0.00003269, the highest frequency of rs559712998 in the East Asian population was 0.002574 , the highest frequency of rs771533925 in the East Asian population was 0.00005568, and the total frequency of rs559712998 mutations was 0.000192; this was the highest frequency of the three mutation sites (Table 5). According to age analysis of the three loci within the global population, we found that the rs 72677250 mutation site was predominant in subjects aged 5055 years, the rs 559712998 mutation site was predominant in subjects aged 30-80 years, and the rs 771533925 mutation site was predominant in subjects aged 65-70 years (Figure 5a,b and c).

\section{The deleterious effects of rs771533925, rs559712998 and rs72677250}

SIFT (http://provean.jcvi.org/index.php) [44] PROVEAN (http://provean.jcvi.org/index.php) [45] and PolyPhen (http://genetics.bwh.harvard.edu/pph2/) [46] algorithms were used to predict the effects of amino acid substitutions on protein function (Table 6). rs771533925 was considered potentially damaging by all three databases, rs559712998 was considered to be tolerated in the above mentioned databases, while rs72677250 was considered to be tolerated in the SIFT database but potentially harmful in the PROVEAN and PolyPhen databases.

\begin{tabular}{|lllll|}
\hline \multicolumn{5}{|c|}{ Table 6 } \\
ID & Hazard prediction of RS771533925, RS559712998 and RS72677250 mutations \\
\hline rs771533925 & TTN & Deleterious & Damaging & possibly_damaging \\
rs559712998 & TTN & Neutral & Tolerated & benign \\
\hline rs72677250 & TTN & Deleterious & Tolerated & possibly_damaging \\
\hline
\end{tabular}

Notes:

PROVEAN (Protein Variation Effect Analyzer) is a tool to predict whether biomolecular structure Variation affects Protein function $\mathbb{S} \mathrm{SIFT}$ (sorts intolerant from tolerant) is a tool for predicting non-synonymous variations based on sequence homology『PolyPhen (Polymorphism Phenotyping ) is a tool which predicts possible impact of an amino acid substitution on the structure and function of a human protein using straightforward physical and comparative considerations.

\section{Functional analysis of TTN}

GO analysis was conducted using Cytoscape 3.8.2 software with the ClueGO [47] plugin. The database (GO Plasmodium falciparum) used for the $\mathrm{GO}$ analysis was acquired from the $\mathrm{GO}$ Consortium. GO analysis was conducted using a two-sided hypergeometric test with Bonferroni correction. The GO term levels were from five to 
ten. The minimum number of genes to form a cluster was set at one. GO analysis showed that the targets for TTN were involved in a range of important biological processes, including myosin thick filament assembly in the skeletal muscle, the positive regulation of protein transport, serine/threonine kinase activity, and cardiac muscle fiber development (Figure 5d).

\section{Validation of candidate loci by CRISPR-Cas12a}

The test results obtained by the CRISPR-Cas12a system for mutation loci in the TTN gene in family samples (Figure 6) were consistent with those obtained from WES sequencing,the presence of mutations in the family samples was verified. We then used the CRISPR-Cas12a system to test a total of 50 sporadic samples for gene mutations. No mutations were found at rs771533925, rs559712998 and rs72677250 of TTN gene in sporadic samples (Figure 7). Therefore, we can conclude that mutations within the TTN locus may play an important role in the pedigree inheritance of MMD. Therefore, this technology is suitable for identifying pedigree genetic patients and assessing the genetic risk of MMD in large-scale screening strategies.

\section{Discussion}

MMD is mostly found in Asia [48]. When considering East Asian countries, such as China, Japan, and Korea, it was found that the highest incidence of MMD was in Japan $(0.54 / 100,000)$ [49]. The clinical manifestations of MMD can vary. According to the different loci involved, MMD can manifest as headache, seizures, syncope, dysarthria, memory loss, personality changes, and other symptoms when the frontal lobe is damaged [50]. In this study, we detected mutant loci in MMD patients and healthy controls to investigate differences in the mutation loci across the population and between regions. We also performed WES on 12 family members of two MMD families to identify MMD genetic-related mutation loci. Finally, we compared CRISPR-cas12a technology with Sanger sequencing and WES for the detection of mutations and highlighted the diagnostic efficacy of CRISPRcas12a.

RNF213 was the first pathogenic gene to be associated with MMD [2]. The R4810K and A4399T mutations in the RNF213 gene are known to be associated with the pathogenesis of MMD in Chinese subjects [10-13]. RNF213 is located on human chromosome 17 (the 17q25.3 region) and is expressed in the liver, lungs, connective tissue, and immune tissue [51]. The main function of MMP3 is to degrade extracellular matrix. MMP3 and its inhibitor are known to regulate the balance between smooth muscle cells and the extracellular matrix. An imbalance leads to vascular smooth muscle hyperplasia and thickening, thus leading to vascular stenosis, one of the key pathogenic factors responsible for MMD [8]. Studies have shown that the $1171(6 \mathrm{~A} / 6 \mathrm{~A})$ mutation in the MMP3 gene is associated with a risk of MMD and that the risk of the (6A/6A) genotype is higher than that of the (5A/6A) genotype [52-54].

In this study, we detected R4810K and A4399T mutations in the RNF213 gene in MMD patients and in the healthy control group. The R4810K mutation was not detected in the healthy control group but was detected at a significantly higher rate in the case group $(P<0.05)$. There was no significant difference in the detection rate for the A4399T locus when compared between the case group and the healthy control group $(P>0.05)$, thus indicating that the R4810K mutation in the RNF213 gene has a potential role in the occurrence and development of MMD and deserves further research attention. 
We also detected an insertion mutation within the MMP3 gene (rs3025058) by first-generation sequencing. The mutation rate of patients with MMD in Shandong Province was 67.6\% compared to 5.4\% in the control group. This compares to a rate of $85 \%$ in the control group from Wuhan City $(\mathrm{P}<0.05)$. Analysis of the MMP3 gene mutation results for the two regions showed that MMD may exhibit regional differences, and that MMP3 could be used as a regional detection index for MMD. These findings should now be confirmed in a larger sample size.

A large number of studies have confirmed that MMD is associated with genetic susceptibility and that genetic factors play a major role in the pathogenesis of this disease [2-4]. In this study, 12 members of two MMD families were sequenced by WES. We identified a number of recessive pathogenic genes. The TTN was identified as the gene containing mutation-related loci (rs771533925, rs559712998 and rs72677250) The Exome Convergence Alliance (EXAC) database was used to analyze the risk alleles (rs72677250, rs559712998 and rs771533925). We identified significant differences in the frequencies of these alleles across the global population. Based on PROVEAN, SIFT, and PolyPhen algorithms, rs771533925 and rs72677250 were considered to be potentially damaging in all three databases while rs559712998 was considered to be tolerated in all three databases. GO analysis showed that the targets of TTN were involved in many important biological processes. The TTN gene encodes myonectin, a protein that is responsible for regulating the passive elasticity of muscle and plays a key role in the development, structural formation, and functional regulation, of the myocardium and skeletal muscle. Together with actin and myosin, the TTN protein constitutes an important component of human cardiac muscle and skeletal muscle $[55,56]$. TTN, as an antigen for the serum antibody of patients with melanoma-associated retinopathy, is a potential biomarker for melanoma and may exert a carcinogenic effect. Future studies should address the role of TTN gene mutation in the pathogenesis of MMD.

The CRISPR-Cas system can recognize foreign DNA or RNA, cause cleavage and silence the expression of the foreign gene [57-59]. This precise targeting ability led directly to the development of the CRISPR-Cas system as an efficient gene editing tool [60-62]. Studies have indicated that a diagnostic platform based on CRISPR-Cas represents an exciting prospect for the detection of cancer and genetic diseases [63]. Cas12a (cpf1) is a new type of programmable DNA enzyme found in the CRISPR system [22]. In the presence of specific directing crRNA, Cas12a also has the ability to cut non-target DNA following cleavage of the target DNA [23, 24]. Therefore, the CRISPR-Cas12a system can be more effective for in vitro detection by adding a fluorescent DNA reporter [64-66] that can emit detectable fluorescence after cleavage. In the present study, we developed CRISPR-Cas12a as a novel assay that could sensitively and specifically detect MMD mutation gene loci. R4810K and A4399T mutations of the RNF213 gene were successfully detected by the CRISPR-cas12a system and first-generation Sanger sequencing with $100 \%$ agreement between the two methods. This indicated that CRISPR-cas $12 a$ technology can be used for the detection of MMD mutations. Compared with first-generation sanger sequencing, the CRISPR-cas12a method is simpler, cheaper, more sensitive, and therefore, should be promoted.

We used CRISPR-cas12a to detect mutations in candidate genes within two families. The results obtained were consistent with those generated by WES, in that SNP loci (rs771533925, rs559712998, and rs72677250) were detected within the TTN gene in pedigree samples. The detection of mutations in the TTN gene in 50 clinical samples revealed that there were no mutations in the TTN gene SNP loci (rs771533925, rs559712998 and rs72677250) and no recessive genetic risk for loci mutations. Therefore, we can conclude that mutations at the TTN locus may play an important role in MMD pedigree inheritance, and is therefore suitable for identifying pedigree genetic patients and assessing the genetic risk of MMD in large-scale screening strategies. 
At present,the genetic screening for MMD disease was limited to the RNF213 gene, or full-gene detection. This was associated with certain limitations, including a long detection time and high costs; furthermore, this was not a convenient strategy for the screening of family samples. Therefore, there is an urgent need for a simple and rapid method to screen for MMD.

\section{Conclusion}

In our study,We found that CRISPR-Cas12a was very effective for detecting MMD mutations and was consistent with WES and sanger sequencing. The turnaround time for the CRISPR-Cas12a system was much shorter than that of next-generation sequencing and WES, implying that this system could be used for the rapid detection of MMD mutations. Furthermore, when using this system, there is no need for large amounts of sample or expensive machines. It is likely that the CRISPR-Cas12a system will be developed as an accurate, portable diagnostic test for the diagnosis and prognosis of MMD. In addition, the CRISPR-Cas12a system only requires a simple and easyto-use microplate reader to provide a fluorescence-based assay for the quantitative measurement of MMD mutations. Therefore, the CRISPR-Cas12a system can overcome the obstacles created by previous platforms and provide a highly sensitive and convenient detection system for MMD mutations using DNA acquired from clinical blood samples. In the future, it will be possible to jointly screen the TTN and RNF213 regions using the CRISPRCas12a system, which not only involves analysis of a patient but could also be extended further to include the lineage to comprehensively assess the genetic risk of familial MMD.

\section{Abbreviations}

MMD: Moyamoya disease

EXAC: Exome Aggregation Consortium

GO: Gene Ontology

SNP: single nucleotide polymorphism

TTN. Titin

WES: whole exome sequencing

GWAS: genome-wide association studies

MAF: minor allele frequency

CT: computed tomography ;MRI:Magnetic resonance imaging

DSA: DigitalSubtractionAngiography

PCR: polymerase chain reaction

crDNA: regions and oligonucleotides

CNVs: Copy number variants 
SNVs: single nucleotide variants

ACMG: American College of Medical Genetics and Genomics

IBD: identity-by-descent

UTR: Untranslated Regions

EXAC: Exome Convergence Alliance

gVCF: Genome VCF

SIFT: Scale Invariant Feature Transform

PAM: protospacer adjacent motif

\section{Declarations}

\section{Ethics approval and consent to participate}

Informed consent was obtained from all patients and the research was approved by the Ethics Committee. of Liaocheng People's Hospital of PLA (No.2020017).

\section{Consent for publication}

Written informed consent for publication was obtained from all participants.

\section{Acknowledgments}

We thank the technical support and guidance provided by Wuhan Bojie Biomedical Science and Technology CO., LTD.

\section{Funding Information}

This work was supported by the National Natural Science Foundation of China [grant number 81701159]; the Latitudinal research project of Shandong University [grant number 6010120011]; the Key Research Project of Shandong University [grant number 2018GSF118046]; the General Program of Postdoctoral Science Foundation of China [grant number 2018M6321231]; the Special Foundation for Postdoctoral Innovation Project of Shandong Province [grant number 201703084]; and the Youth Program of National Natural Science Foundation of Shandong Province [grant number ZR2015HQ023]; and the Taishan Scholar Project of Shandong Province of China(no.tsqn202103200) .

\section{Availability of data and materials}




\section{Conflict of interest}

The authors declare that they have no conflict of interest.

\section{Author contributions}

Yilei Xiao, Weidong Liu and Jiheng Hao conceived the experiments. Yuejiu Pang, Qunlong Jiang, Min Zhang, Xingbang Wang, Xueyuan Li and Zixin Guo conducted the experiments. Donghu Yu, Xiaoping Liu, Jie Yao, Fangang Meng, Liyong Zhang, Zhaogang Dong and Jiyue Wang analyzed the results. All authors reviewed the manuscript.

\section{References}

1. Kuroda S, Houkin K: Moyamoya disease: current concepts and future perspectives. Lancet Neurol 2008, 7(11):1056-1066.

2. Miao W, Zhao PL, Zhang YS, Liu HY, Chang Y, Ma J, Huang QJ, Lou ZX: Epidemiological and clinical features of Moyamoya disease in Nanjing, China. Clin Neurol Neurosurg 2010, 112(3):199-203.

3. Acker G, Goerdes S, Schneider UC, Schmiedek P, Czabanka M, Vajkoczy P: Distinct clinical and radiographic characteristics of moyamoya disease amongst European Caucasians. Eur J Neurol 2015, 22(6):1012-1017.

4. Kamada F, Aoki Y, Narisawa A, Abe Y, Komatsuzaki S, Kikuchi A, Kanno J, Niihori T, Ono M, Ishii N et al: A genome-wide association study identifies RNF213 as the first Moyamoya disease gene. J Hum Genet 2011, 56(1):34-40.

5. Wu Z, Jiang H, Zhang L, Xu X, Zhang X, Kang Z, Song D, Zhang J, Guan M, Gu Y: Molecular analysis of RNF213 gene for moyamoya disease in the Chinese Han population. PLoS One 2012, 7(10):e48179.

6. Zhang Q, Liu Y, Zhang D, Wang R, Zhang Y, Wang S, Yu L, Lu C, Liu F, Zhou J et al: RNF213 as the major susceptibility gene for Chinese patients with moyamoya disease and its clinical relevance. J Neurosurg 2017, 126(4):1106-1113.

7. Wang Y, Zhang Z, Wei L, Zhang Q, Zou Z, Yang L, Li D, Shang M, Han C, Mambiya M et al: Predictive role of heterozygous p.R4810K of RNF213 in the phenotype of Chinese moyamoya disease. Neurology 2020, 94(7):e678-e686.

8. Li H, Zhang ZS, Liu W, Yang WZ, Dong ZN, Ma MJ, Han C, Yang H, Cao WC, Duan L: Association of a functional polymorphism in the MMP-3 gene with Moyamoya Disease in the Chinese Han population. Cerebrovasc Dis 2010, 30(6):618-625.

9. Kim JS: Moyamoya Disease: Epidemiology, Clinical Features, and Diagnosis. Journal of stroke 2016, 18(1):211.

10. Morito D, Nishikawa K, Hoseki J, Kitamura A, Kotani Y, Kiso K, Kinjo M, Fujiyoshi Y, Nagata K: Moyamoya disease-associated protein mysterin/RNF213 is a novel AAA+ ATPase, which dynamically changes its oligomeric state. Sci Rep 2014, 4:4442. 
11. Kobayashi H, Matsuda Y, Hitomi T, Okuda H, Shioi H, Matsuda T, Imai H, Sone M, Taura D, Harada KH et al: Biochemical and Functional Characterization of RNF213 (Mysterin) R4810K, a Susceptibility Mutation of Moyamoya Disease, in Angiogenesis In Vitro and In Vivo. Journal of the American Heart Association 2015, 4(7).

12. Liu W, Morito D, Takashima S, Mineharu Y, Kobayashi H, Hitomi T, Hashikata H, Matsuura N, Yamazaki S, Toyoda A et al: Identification of RNF213 as a susceptibility gene for moyamoya disease and its possible role in vascular development. PLoS One 2011, 6(7):e22542.

13. Hu J, Luo J, Chen Q: The Susceptibility Pathogenesis of Moyamoya Disease. World Neurosurg 2017, 101:731-741.

14. Ikezaki K, Inamura T, Kawano T, Fukui M: Clinical features of probable moyamoya disease in Japan. Clin Neurol Neurosurg 1997, 99 Suppl 2:S173-177.

15. Hervé D, Touraine P, Verloes A, Miskinyte S, Krivosic V, Logeart D, Alili N, Laredo JD, Gaudric A, Houdart E et al: A hereditary moyamoya syndrome with multisystemic manifestations. Neurology 2010, 75(3):259-264.

16. Han C, Feng H, Han YQ, Liu WW, Zhang ZS, Yang WZ, Duan L: Prospective screening of family members with moyamoya disease patients. PLoS One 2014, 9(2):e88765.

17. Ahn HS, Kazmi SZ, Kang T, Kim DS, Ryu T, Oh JS, Hann HJ, Kim HJ: Familial Risk for Moyamoya Disease Among First-Degree Relatives, Based on a Population-Based Aggregation Study in Korea. Stroke 2020, 51(9):2752-2760.

18. Hishikawa T, Tokunaga K, Sugiu K, Date I: Clinical and radiographic features of moyamoya disease in patients with both cerebral ischaemia and haemorrhage. Br J Neurosurg 2013, 27(2):198-201.

19. Ishino Y, Shinagawa H, Makino K, Amemura M, Nakata A: Nucleotide sequence of the iap gene, responsible for alkaline phosphatase isozyme conversion in Escherichia coli, and identification of the gene product. $J$ Bacteriol 1987, 169(12):5429-5433.

20. Jansen R, Embden JD, Gaastra W, Schouls LM: Identification of genes that are associated with DNA repeats in prokaryotes. Mol Microbiol 2002, 43(6):1565-1575.

21. Mojica FJ, Díez-Villaseñor C, García-Martínez J, Soria E: Intervening sequences of regularly spaced prokaryotic repeats derive from foreign genetic elements. J Mol Evol 2005, 60(2):174-182.

22. Zhou Y, Zhu S, Cai C, Yuan P, Li C, Huang Y, Wei W: High-throughput screening of a CRISPR/Cas9 library for functional genomics in human cells. Nature 2014, 509(7501):487-491.

23. Qi LS, Larson MH, Gilbert LA, Doudna JA, Weissman JS, Arkin AP, Lim WA: Repurposing CRISPR as an RNAguided platform for sequence-specific control of gene expression. Cell 2013, 152(5):1173-1183.

24. Gilbert LA, Larson MH, Morsut L, Liu Z, Brar GA, Torres SE, Stern-Ginossar N, Brandman O, Whitehead EH, Doudna JA et al: CRISPR-mediated modular RNA-guided regulation of transcription in eukaryotes. Cell 2013, 154(2):442-451.

25. Chertow DS: Next-generation diagnostics with CRISPR. Science 2018, 360(6387):381-382.

26. McClellan JM, Susser E, King MC: Schizophrenia: a common disease caused by multiple rare alleles. $\mathrm{Br} J$ Psychiatry 2007, 190:194-199.

27. McClellan J, King MC: Genetic heterogeneity in human disease. Cell 2010, 141(2):210-217.

28. Manolio TA, Collins FS, Cox NJ, Goldstein DB, Hindorff LA, Hunter DJ, McCarthy MI, Ramos EM, Cardon LR, Chakravarti A et al: Finding the missing heritability of complex diseases. Nature 2009, 461(7265):747-753. 
29. Tu M, Lin L, Cheng Y, He X, Sun H, Xie H, Fu J, Liu C, Li J, Chen D et al: A 'new lease of life': FnCpf1 possesses DNA cleavage activity for genome editing in human cells. Nucleic Acids Res 2017, 45(19):11295-11304.

30. Li H, Durbin R: Fast and accurate short read alignment with Burrows-Wheeler transform. Bioinformatics 2009, 25(14):1754-1760.

31. Tarasov A, Vilella AJ, Cuppen E, Nijman IJ, Prins P: Sambamba: fast processing of NGS alignment formats. Bioinformatics 2015, 31(12):2032-2034.

32. Li H, Handsaker B, Wysoker A, Fennell T, Ruan J, Homer N, Marth G, Abecasis G, Durbin R: The Sequence Alignment/Map format and SAMtools. Bioinformatics 2009, 25(16):2078-2079.

33. Krumm N, Sudmant PH, Ko A, O'Roak BJ, Malig M, Coe BP, Quinlan AR, Nickerson DA, Eichler EE: Copy number variation detection and genotyping from exome sequence data. Genome Res 2012, 22(8):1525-1532.

34. Wang K, Li M, Hakonarson H: ANNOVAR: functional annotation of genetic variants from high-throughput sequencing data. Nucleic Acids Res 2010, 38(16):e164.

35. Auton A, Brooks LD, Durbin RM, Garrison EP, Kang HM, Korbel JO, Marchini JL, McCarthy S, McVean GA, Abecasis GR: A global reference for human genetic variation. Nature 2015, 526(7571):68-74.

36. Kumar P, Henikoff S, Ng PC: Predicting the effects of coding non-synonymous variants on protein function using the SIFT algorithm. Nat Protoc 2009, 4(7):1073-1081.

37. Adzhubei IA, Schmidt S, Peshkin L, Ramensky VE, Gerasimova A, Bork P, Kondrashov AS, Sunyaev SR: A method and server for predicting damaging missense mutations. Nature methods 2010, 7(4):248-249.

38. Schwarz JM, Rödelsperger C, Schuelke M, Seelow D: MutationTaster evaluates disease-causing potential of sequence alterations. Nature methods 2010, 7(8):575-576.

39. Kircher M, Witten DM, Jain P, O'Roak BJ, Cooper GM, Shendure J: A general framework for estimating the relative pathogenicity of human genetic variants. Nat Genet 2014, 46(3):310-315.

40. Muona M, Berkovic SF, Dibbens LM, Oliver KL, Maljevic S, Bayly MA, Joensuu T, Canafoglia L, Franceschetti $\mathrm{S}$, Michelucci R et al: A recurrent de novo mutation in KCNC1 causes progressive myoclonus epilepsy. Nat Genet 2015, 47(1):39-46.

41. Richards S, Aziz N, Bale S, Bick D, Das S, Gastier-Foster J, Grody WW, Hegde M, Lyon E, Spector E et al: Standards and guidelines for the interpretation of sequence variants: a joint consensus recommendation of the American College of Medical Genetics and Genomics and the Association for Molecular Pathology. Genet Med 2015, 17(5):405-424.

42. Purcell S, Neale B, Todd-Brown K, Thomas L, Ferreira MA, Bender D, Maller J, Sklar P, de Bakker PI, Daly MJ et al: PLINK: a tool set for whole-genome association and population-based linkage analyses. Am J Hum Genet 2007, 81(3):559-575.

43. Lek M, Karczewski KJ, Minikel EV, Samocha KE, Banks E, Fennell T, O'Donnell-Luria AH, Ware JS, Hill AJ, Cummings BB et al: Analysis of protein-coding genetic variation in 60,706 humans. Nature 2016, 536(7616):285-291.

44. Choi Y, Chan AP: PROVEAN web server: a tool to predict the functional effect of amino acid substitutions and indels. Bioinformatics 2015, 31(16):2745-2747.

45. Vaser R, Adusumalli S, Leng SN, Sikic M, Ng PC: SIFT missense predictions for genomes. Nat Protoc 2016, 11(1):1-9. 
46. Adzhubei I, Jordan DM, Sunyaev SR: Predicting functional effect of human missense mutations using PolyPhen-2. Current protocols in human genetics 2013, Chapter 7:Unit7.20.

47. Bindea G, Mlecnik B, Hackl H, Charoentong P, Tosolini M, Kirilovsky A, Fridman WH, Pagès F, Trajanoski Z, Galon J: ClueGO: a Cytoscape plug-in to decipher functionally grouped gene ontology and pathway annotation networks. Bioinformatics 2009, 25(8):1091-1093.

48. Kamimura T, Okazaki S, Morimoto T, Kobayashi H, Harada K, Tomita T, Higashiyama A, Yoshimoto T, Takahashi JC, Nakagawara J et al: Prevalence of RNF213 p.R4810K Variant in Early-Onset Stroke With Intracranial Arterial Stenosis. Stroke 2019, 50(6):1561-1563.

49. Miyatake S, Miyake N, Touho H, Nishimura-Tadaki A, Kondo Y, Okada I, Tsurusaki Y, Doi H, Sakai H, Saitsu H et al: Homozygous c.14576G>A variant of RNF213 predicts early-onset and severe form of moyamoya disease. Neurology 2012, 78(11):803-810.

50. Baba T, Houkin K, Kuroda S: Novel epidemiological features of moyamoya disease. J Neurol Neurosurg Psychiatry 2008, 79(8):900-904.

51. Kuriyama S, Kusaka Y, Fujimura M, Wakai K, Tamakoshi A, Hashimoto S, Tsuji I, Inaba Y, Yoshimoto T: Prevalence and clinicoepidemiological features of moyamoya disease in Japan: findings from a nationwide epidemiological survey. Stroke 2008, 39(1):42-47.

52. Wang X, Zhang Z, Liu W, Xiong Y, Sun W, Huang X, Jiang Y, Ni G, Sun W, Zhou L et al: Impacts and interactions of PDGFRB, MMP-3, TIMP-2, and RNF213 polymorphisms on the risk of Moyamoya disease in Han Chinese human subjects. Gene 2013, 526(2):437-442.

53. Wang X, Wang Y, Nie F, Li Q, Zhang K, Liu M, Yang L, Zhang Q, Liu S, Zeng F et al: Association of Genetic Variants With Moyamoya Disease in 13000 Individuals: A Meta-Analysis. Stroke 2020, 51(6):1647-1655.

54. Ma J, You C: Association between matrix metalloproteinase-3 gene polymorphism and moyamoya disease. $J$ Clin Neurosci 2015, 22(3):479-482.

55. Vikhlyantsev IM, Podlubnaya ZA: New titin (connectin) isoforms and their functional role in striated muscles of mammals: facts and suppositions. Biochemistry (Mosc) 2012, 77(13):1515-1535.

56. Pföhler C, Preuss KD, Tilgen W, Stark A, Regitz E, Fadle N, Pfreundschuh M: Mitofilin and titin as target antigens in melanoma-associated retinopathy. Int J Cancer 2007, 120(4):788-795.

57. Brouns SJ, Jore MM, Lundgren M, Westra ER, Slijkhuis RJ, Snijders AP, Dickman MJ, Makarova KS, Koonin EV, van der Oost J: Small CRISPR RNAs guide antiviral defense in prokaryotes. Science 2008, 321(5891):960964.

58. Marraffini LA, Sontheimer EJ: CRISPR interference limits horizontal gene transfer in staphylococci by targeting DNA. Science 2008, 322(5909):1843-1845.

59. Garneau JE, Dupuis M, Villion M, Romero DA, Barrangou R, Boyaval P, Fremaux C, Horvath P, Magadán AH, Moineau S: The CRISPR/Cas bacterial immune system cleaves bacteriophage and plasmid DNA. Nature 2010, 468(7320):67-71.

60. Deltcheva E, Chylinski K, Sharma CM, Gonzales K, Chao Y, Pirzada ZA, Eckert MR, Vogel J, Charpentier E: CRISPR RNA maturation by trans-encoded small RNA and host factor RNase III. Nature 2011, 471(7340):602607.

61. Jinek M, Chylinski K, Fonfara I, Hauer M, Doudna JA, Charpentier E: A programmable dual-RNA-guided DNA endonuclease in adaptive bacterial immunity. Science 2012, 337(6096):816-821. 
62. Cong L, Ran FA, Cox D, Lin S, Barretto R, Habib N, Hsu PD, Wu X, Jiang W, Marraffini LA et al: Multiplex genome engineering using CRISPR/Cas systems. Science 2013, 339(6121):819-823.

63. Mali P, Yang L, Esvelt KM, Aach J, Guell M, DiCarlo JE, Norville JE, Church GM: RNA-guided human genome engineering via Cas9. Science 2013, 339(6121):823-826.

64. Nelles DA, Fang MY, O'Connell MR, Xu JL, Markmiller SJ, Doudna JA, Yeo GW: Programmable RNA Tracking in Live Cells with CRISPR/Cas9. Cell 2016, 165(2):488-496.

65. Mohanraju P, Makarova KS, Zetsche B, Zhang F, Koonin EV, van der Oost J: Diverse evolutionary roots and mechanistic variations of the CRISPR-Cas systems. Science 2016, 353(6299):aad5147.

66. Koonin EV, Makarova KS, Zhang F: Diversity, classification and evolution of CRISPR-Cas systems. Curr Opin Microbiol 2017, 37:67-78.

\section{Tables}

Due to technical limitations, table 4 and table 5 are only available as a download in the Supplemental Files section.

\section{Figures}

a

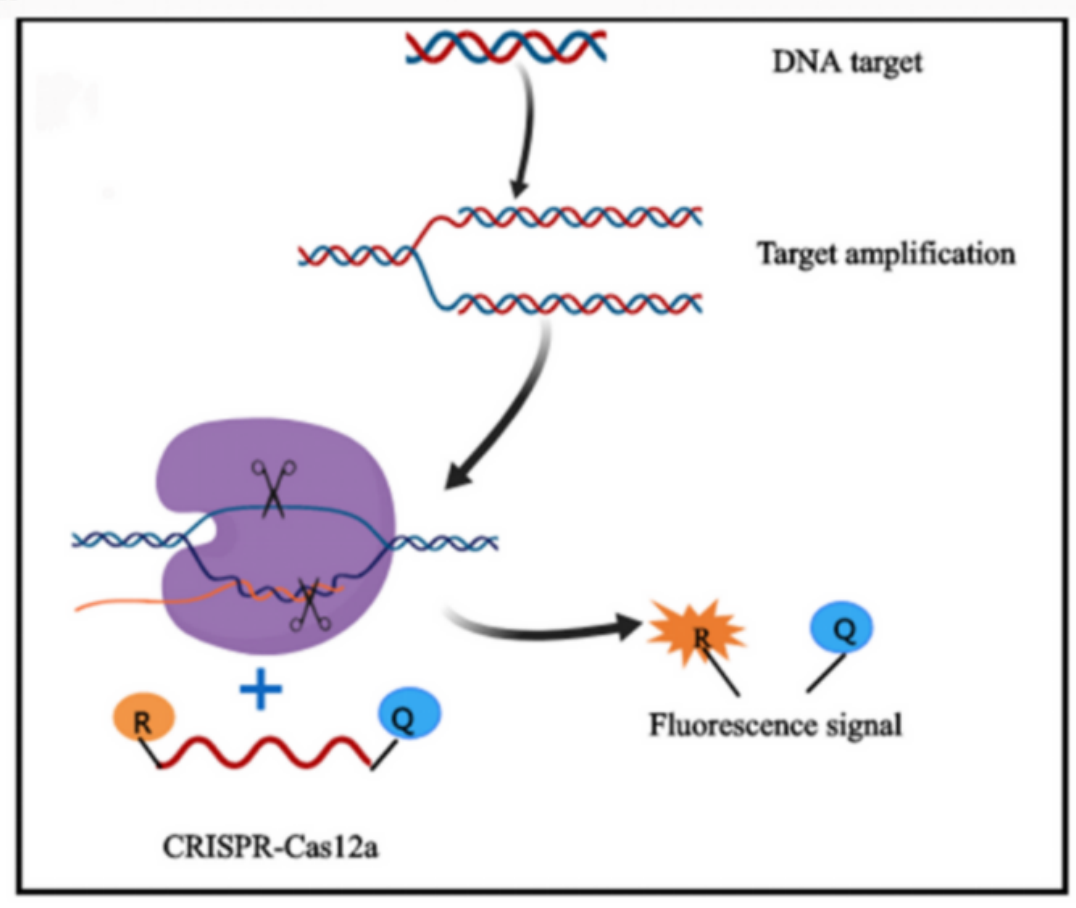

b

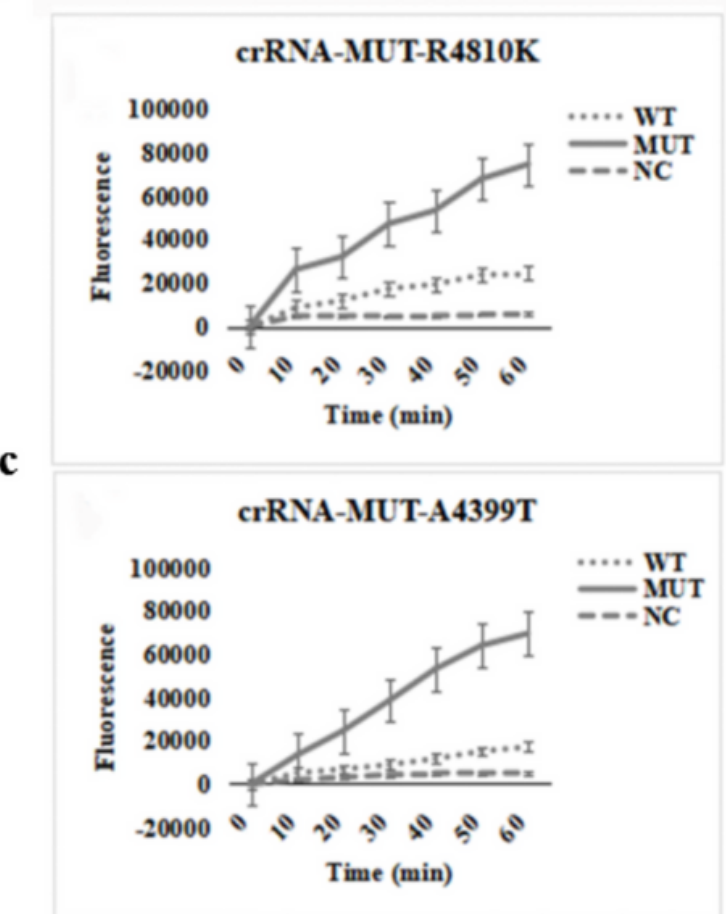

\section{Figure 1}

Validation of the CRISPR-Cas12a detection system (a)The principles underlying CRISPR-cas12a as a detection system; (b and c) Validation of the CRISPR-Cas12a detection system. The fluorescence of Mutant type was significantly higher than that of wild type, and the number of mutant type was more than 2 times of wild type. 
RNF213

a

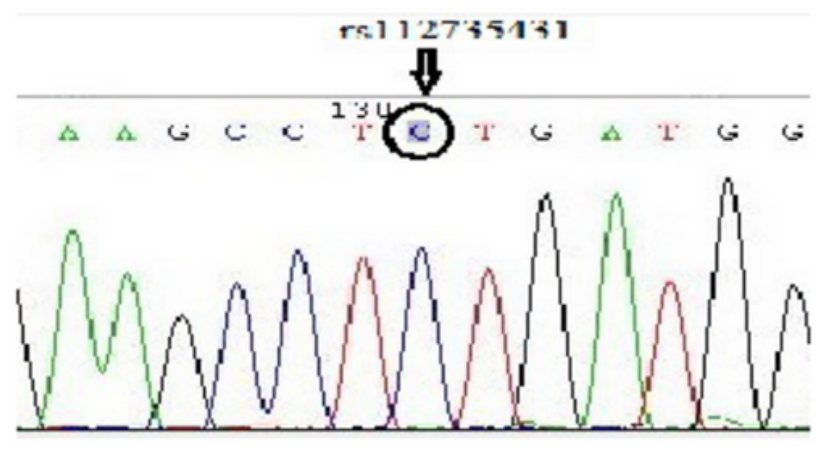

rs 148731719

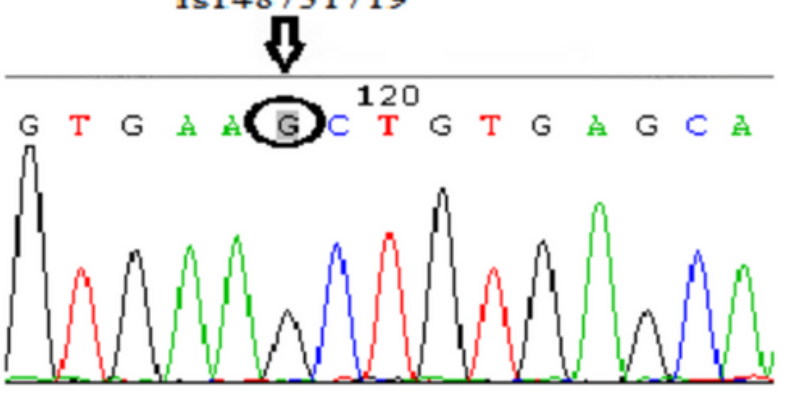

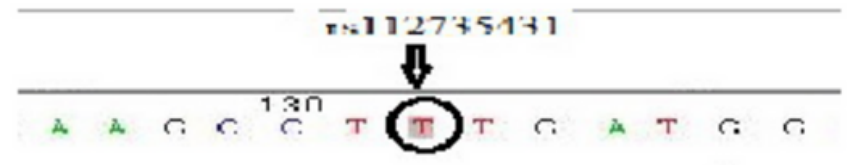

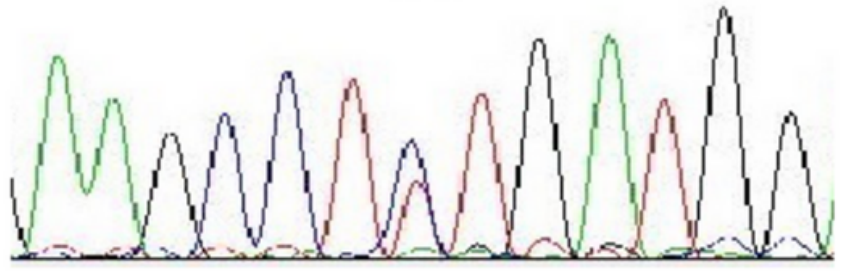

rs 148731719

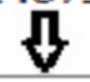

$G T G \Lambda A @ T{ }^{120} T G A G C A$

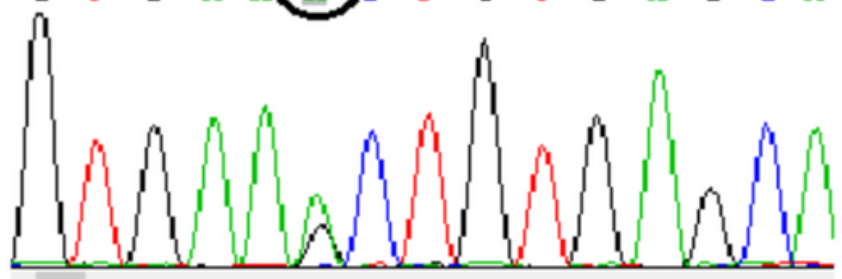

MMP3

b

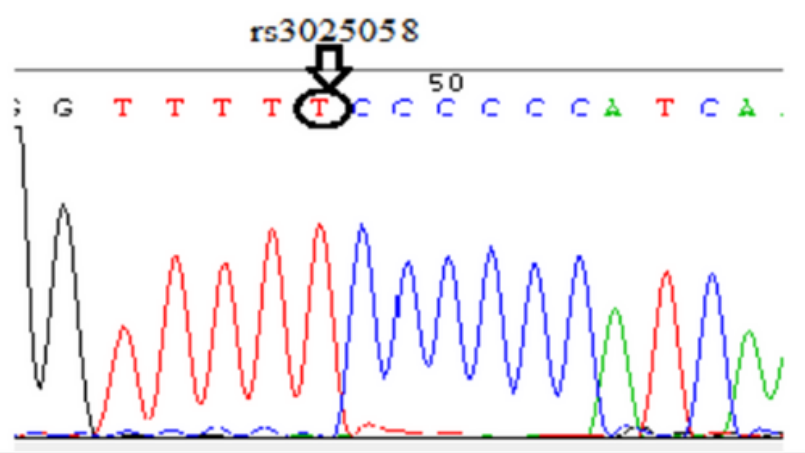

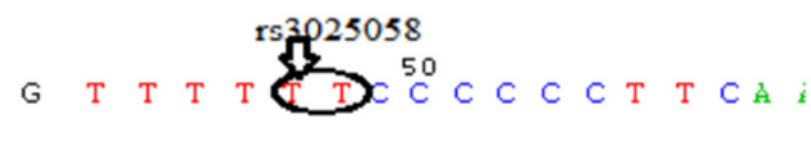

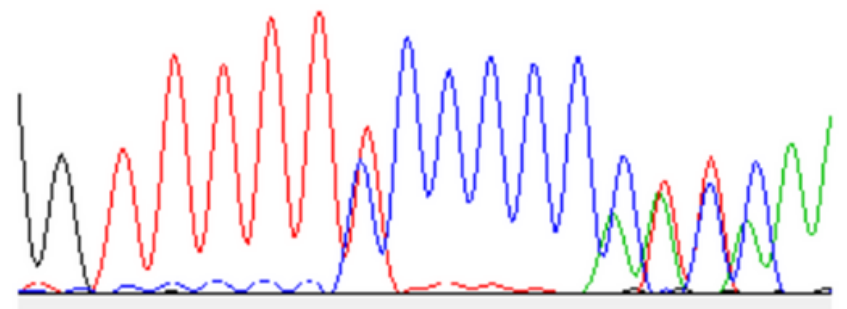

Figure 2

Sequencing Peak Map of RNF213 and MMP3 Gene (a)Sequencing peak map of RNF213 Gene; (b)Sequencing peak map of MMP3 Gene 
Family A

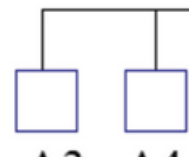

A3 A4
Patients with

MMD: A1, A2 c

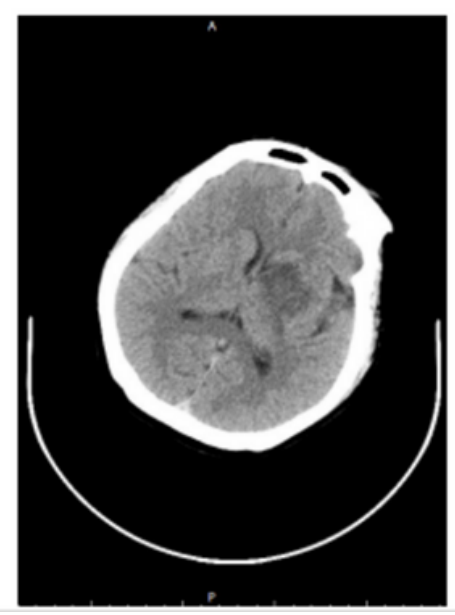

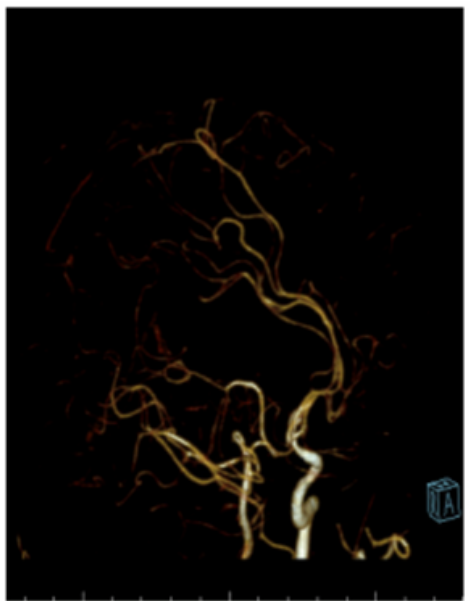

d

Family B

B4

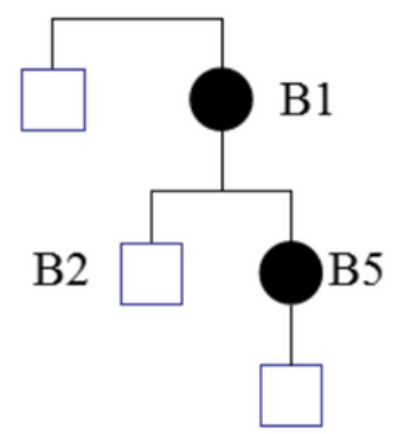

B3
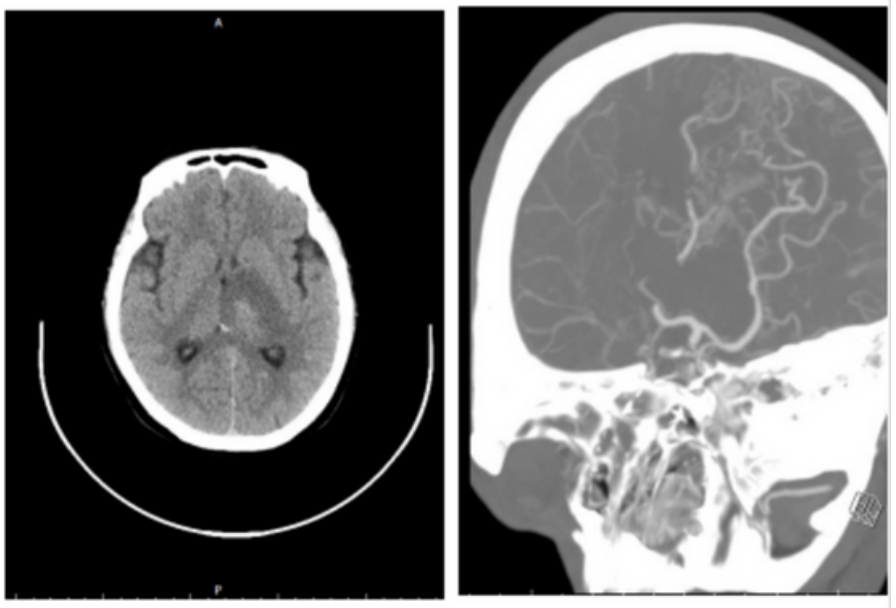

\section{Figure 3}

Exome sequencing maps for the two MMD families. (a and b) Pedigree charts. Squares: male; circles: female; black-filled symbols: patients; (c) CT and CTA of patient A1; (d) CT and CTA of patient B1. 


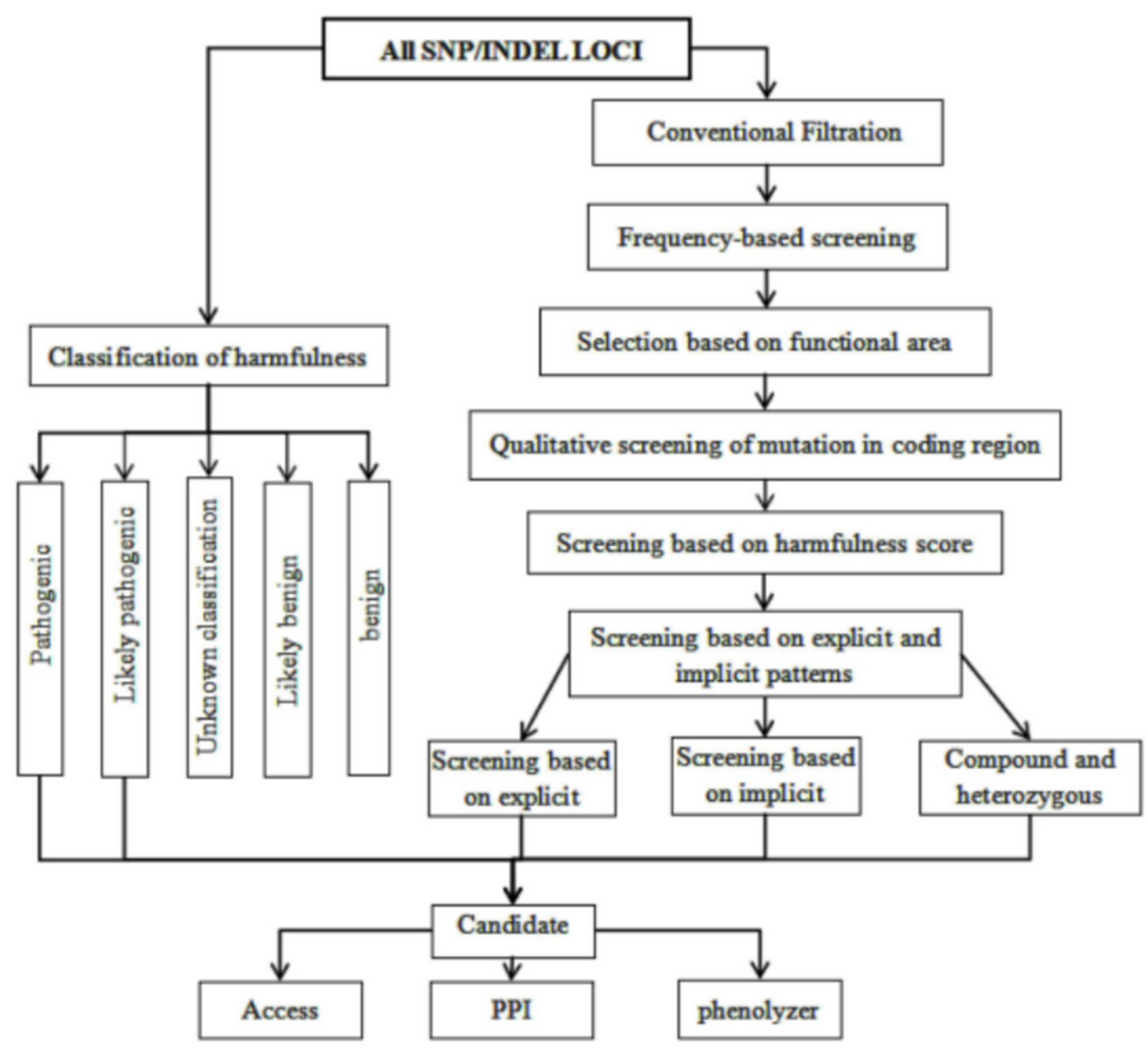

Figure 4

Analysis Flow Chart 2 The main process of advanced analysis:1)The main process of advanced analysis 1 Screening based on mutation sites and their harmfulness ;2) Screening based on sample recessive patterns ;3)Screening based on candidate genes and relationship with disease phenotypes ;4)Pathway enrichment of candidate genes through GO and KEGG Set analysis also uses DisGeNet and Phenolyzer to analyze gene-disease phenotype associations 
a

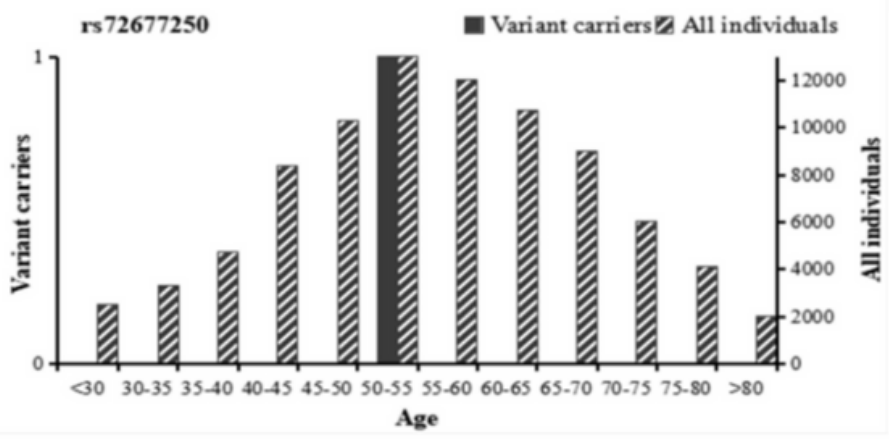

c

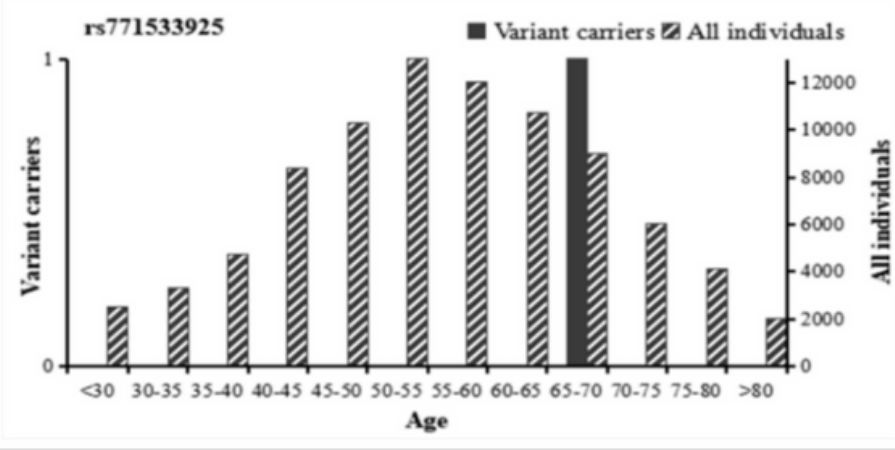

b

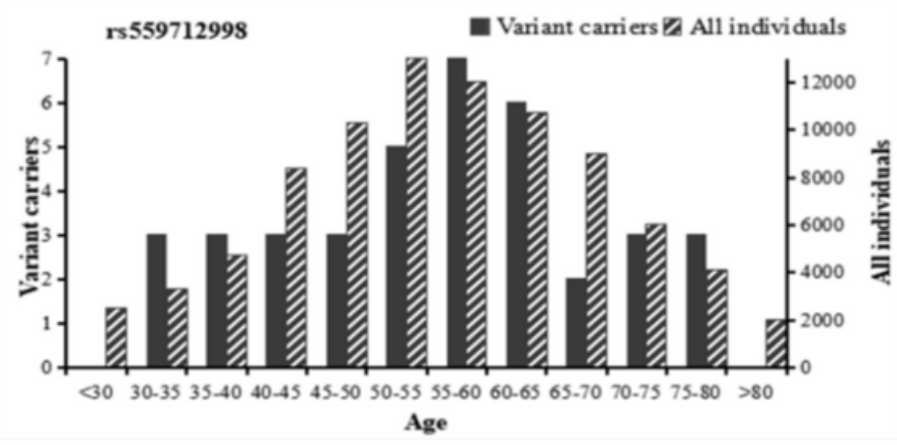

d

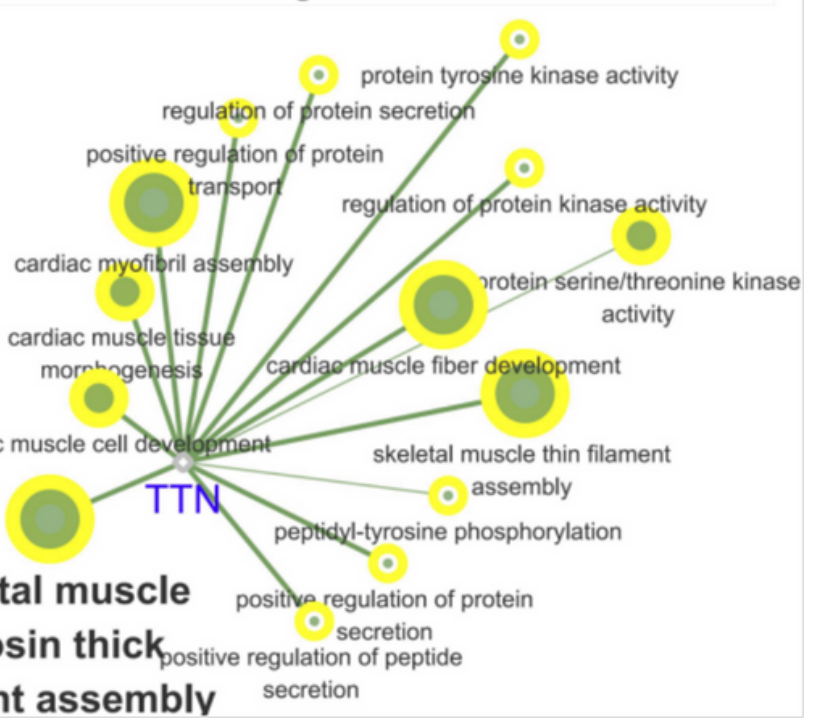

\section{Figure 5}

The frequencies of rs72677250, rs559712998, and rs771533925 mutations in subjects of different ages within the global population, as determined by exome sequencing (a) rs72677250 site mutations in different age groups within the global population; (b) rs559712998 site mutations in different age groups within the global population; (c) rs771533925 site mutations in different age groups within the global population. Analysis included heterozygous variant carriers, homozygous variant carriers. (d)Functional enrichment analysis of TTN,GO analysis was conducted using Cytoscape 3.8.2 software with the ClueGO plugin. GO analysis was conducted using a two-sided hypergeometric test with Bonferroni correction 
a

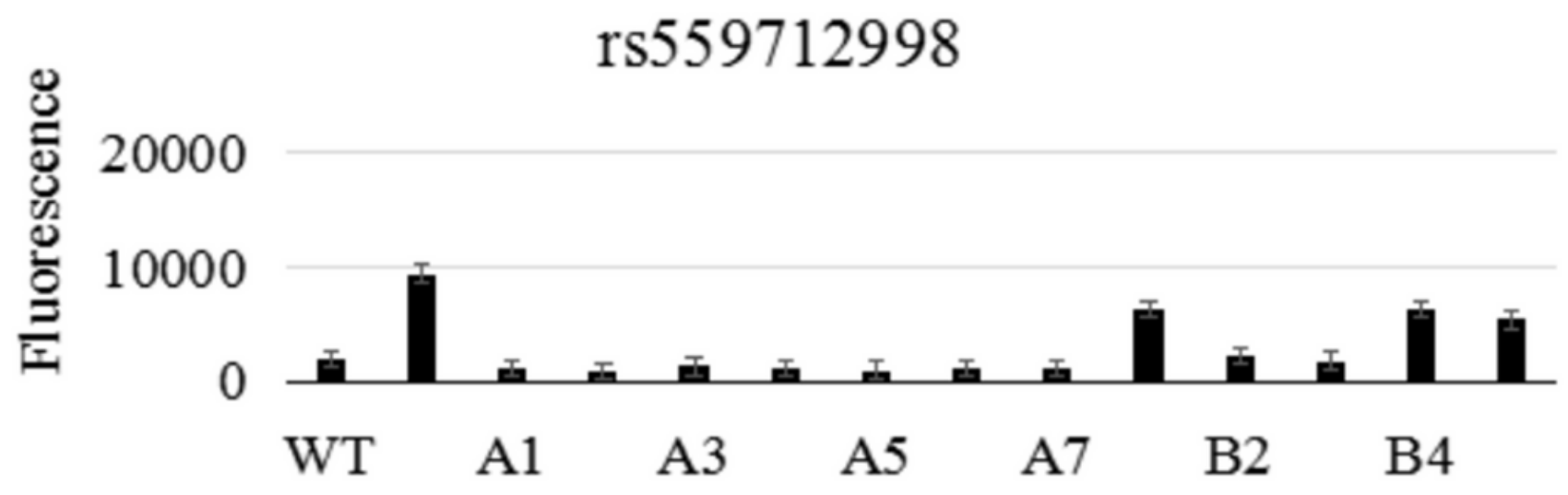

b

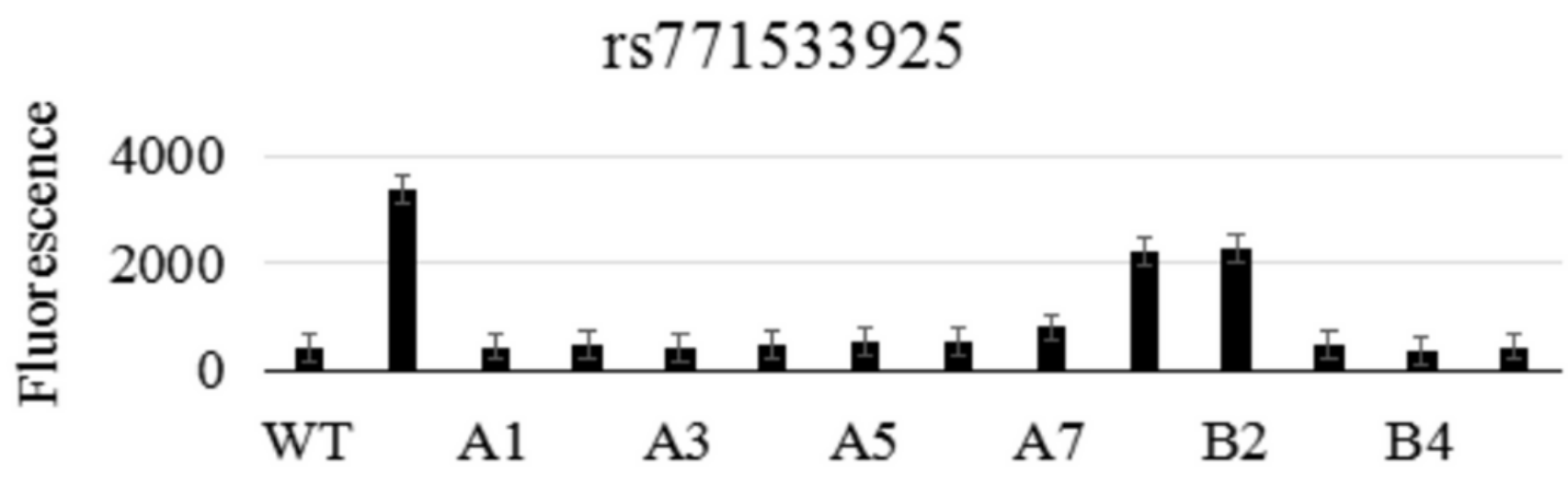

c

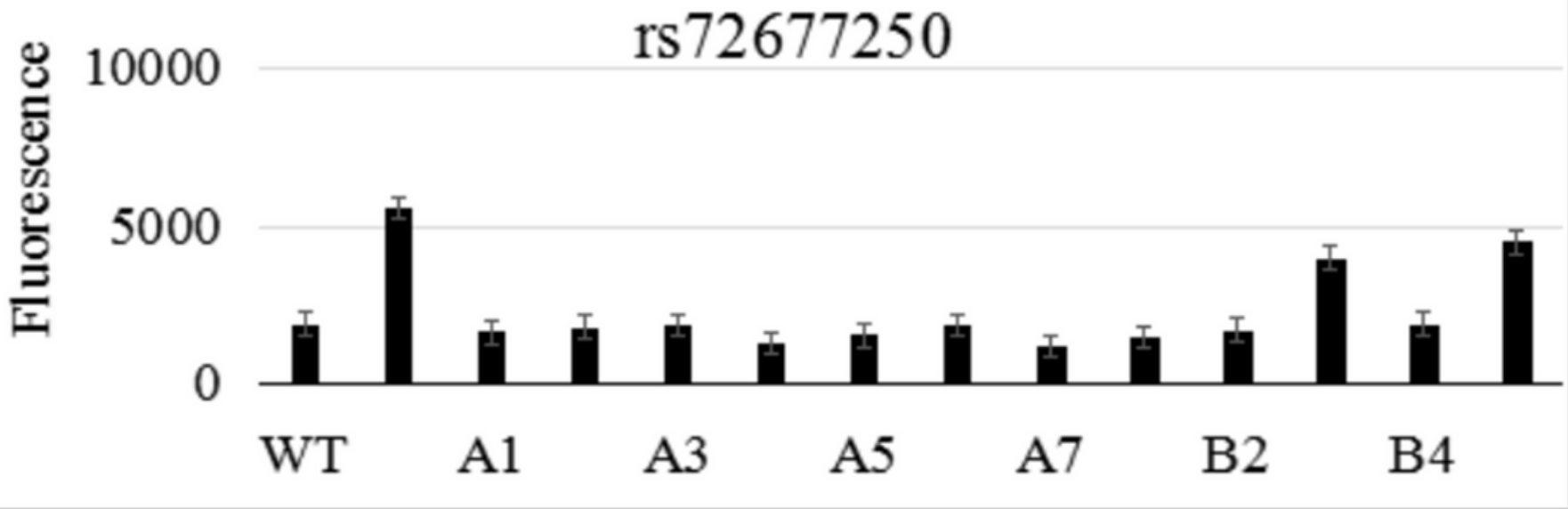

Figure 6

CRISPR-Cas12a test results for mutation loci in the TTN gene in family Samples (a) CRISPR-Cas12a test results for rs72677250 in family samples; (b) CRISPR-Cas12a test results for rs559712998 in family samples; (c) CRISPR-Cas12a test results for rs771533925 in family samples. 


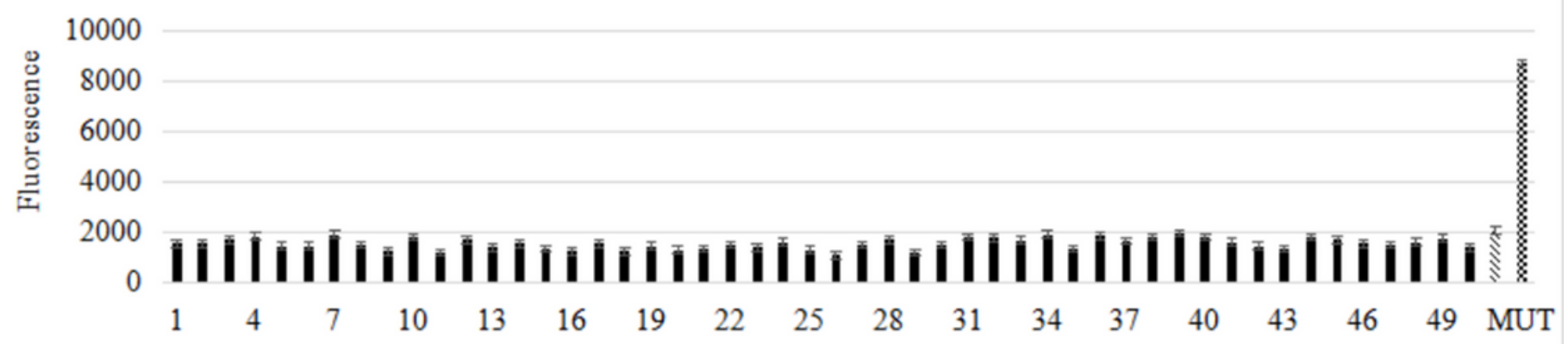

b

rs771533925

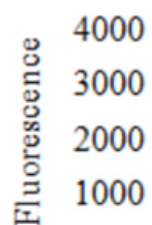

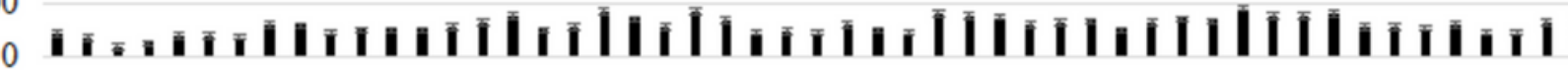

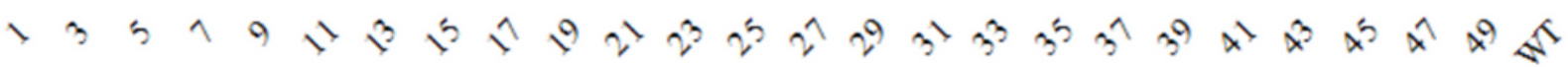

c

rs72677250

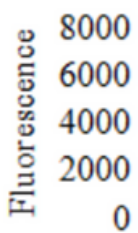

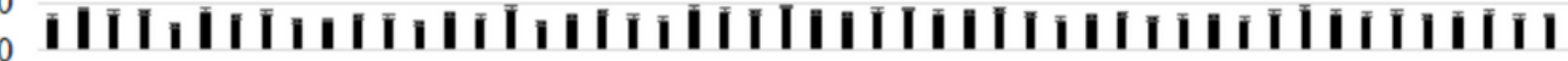

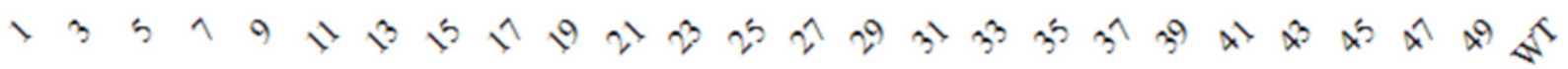

Figure 7

CRISPR-Cas12a test results for mutation loci in the TTN gene in Sporadic Samples (a) CRISPR-Cas12a test results for rs72677250 in sporadic samples; (b) CRISPR-Cas12a test results for rs559712998 in sporadic samples; (c) CRISPR-Cas12a test results for rs771533925 in sporadic samples.

\section{Supplementary Files}

This is a list of supplementary files associated with this preprint. Click to download.

- FigureS1.tif

- Tables1.docx

- Tables2.docx

- Tables3.docx

- Tables4.docx 
- Table5.docx

- Table4.docx 